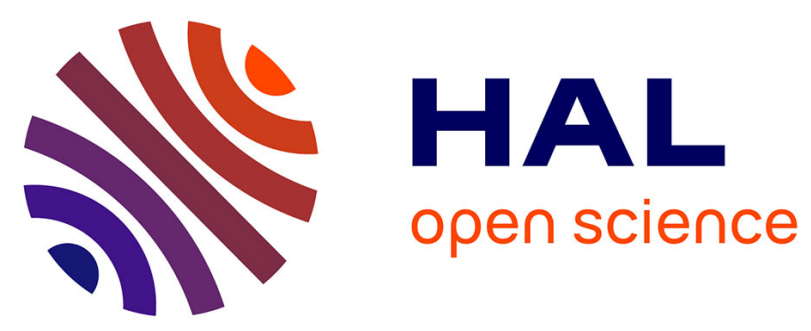

\title{
Integrating prior Knowledge in Weighted SVM for Human Activity Recognition in Smart Home
}

\author{
M 'Hamed Bilal Abidine, Belkacem Fergani, Anthony Fleury
}

\section{To cite this version:}

M 'Hamed Bilal Abidine, Belkacem Fergani, Anthony Fleury. Integrating prior Knowledge in Weighted SVM for Human Activity Recognition in Smart Home. ICOST 2017 - 15th International Conference On Smart homes and health Telematics. IoT for Enhanced Quality of Life and Smart Living, Aug 2017, Marne la vallée, France. hal-01855154

\section{HAL Id: hal-01855154 https://hal.science/hal-01855154}

Submitted on 7 Aug 2018

HAL is a multi-disciplinary open access archive for the deposit and dissemination of scientific research documents, whether they are published or not. The documents may come from teaching and research institutions in France or abroad, or from public or private research centers.
L'archive ouverte pluridisciplinaire HAL, est destinée au dépôt et à la diffusion de documents scientifiques de niveau recherche, publiés ou non, émanant des établissements d'enseignement et de recherche français ou étrangers, des laboratoires publics ou privés. 


\title{
Integrating prior Knowledge in Weighted SVM for Human Activity Recognition in Smart Home
}

\author{
M'hamed Bilal ABIDINE1', Belkacem FERGANI ${ }^{1}$, and Anthony FLEURY ${ }^{2}$ \\ ${ }^{1}$ Laboratoire d'Ingénierie des Systèmes Intelligents et Communicants, LISIC Lab., \\ Electronics and Computer Sciences Dept., University of Science and Technology Houari \\ Boumediene (USTHB), 32, El Alia, Bab Ezzouar, 16111 Algiers, Algeria \\ ${ }^{2}$ Mines Douai, URIA, Douai, France and University of Lille, Lille, France \\ abidineb@hotmail.com
}

\begin{abstract}
Feature extraction and classification are two key steps for activity recognition in a smart home environment. In this work, we performed a new hybrid model using Temporal or Spatial Features (TF or SF) with the PCALDA-WSVM classifier. The last method combines two methods for feature extraction: Principal Component Analysis (PCA), and Linear Discriminant Analysis (LDA) followed by Weighted SVM Classifier. This classifier is used to handle the problem of imbalanced activity data from sensor readings. The experiments that were implemented on multiple real-world datasets, showed the effectiveness of TF and SF attributes combined with PCA-LDA-WSVM in activity recognition.
\end{abstract}

Keywords: Activity recognition; Feature extraction; PCA; LDA; Weighted SVM.

\section{Introduction}

Several classification algorithms have been employed for Human Activity Recognition (HAR) tasks [1, 2, 3, 4] to automatically recognize activities in intelligent manner about the occupants and ensure the comfort of older adults in smart home using sensor networks. In [5], we have developed a new classification method named PCA-LDA-WSVM based on a combination of Principal Component Analysis (PCA), Linear Discriminant Analysis (LDA) and the modified Weighted Support Vector Machines (WSVM). We demonstrated the ability of this method to achieve good improvement over the standard used methods such as HMM, CRF, SVM, WSVM.

Classifiers address the challenge of extracting information from raw sensor data through the use of features. In this paper, we wanted to improve the classification performances of the approach in [5] by introducing prior knowledge [6]. The 'Prepare breakfast' and 'Prepare dinner' activities share the same model as they involve the same set of object interactions. These two activities are distinguished by time of taking place, i.e. 'Prepare breakfast' takes place in the morning hours and "Prepare dinner" takes place in the afternoon or evening hours of the day. The location attribute can also discriminate between two different activity classes as 'Toileting' and 'Showering' that performed in two different locations. 


\section{The Proposed HAR system by introducing the prior knowledge}

\subsection{Overview}

The core idea of proposed method is as follows: A dataset is divided into training and testing sets. Having defined the activities to recognize and the list of potentially interesting features, we added both temporal and spatial features (TF and SF). We then extract the features that proved to be the most useful for activity recognition. These two sets are transformed independently with PCA and LDA methods. By adding those PCF it is possible to have more than the number of classes minus one extracted features by LDA. Then WSVM method, as the latter process as follow.

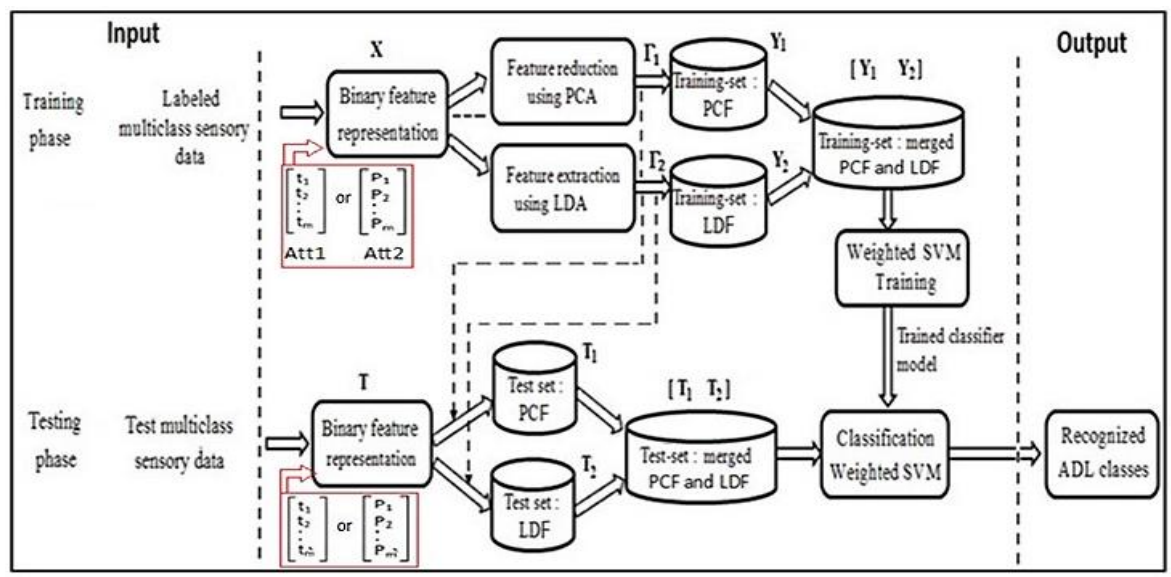

Figure 1. Block diagram of the proposed activity recognition approach using the feature insertion. Att $1=\mathrm{TF}, \mathrm{Att} 2=\mathrm{SF}$.

\subsection{Feature Representation}

Sensors outputs are binary and represented in a feature space which is used by the model to recognize the activities performed. The raw data obtained from the sensors can either be used directly, or be transformed into a different representation forms (Fig. 2). We do not use the raw sensor data representation as observations; instead we use the "Change point" and "Last" representations which have been shown to give much better results in activity recognition [7].

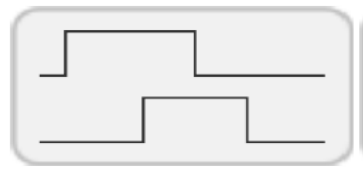

(a) Raw

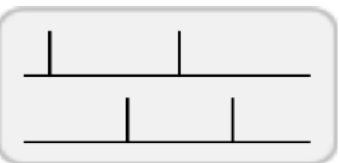

(b) Changepoint

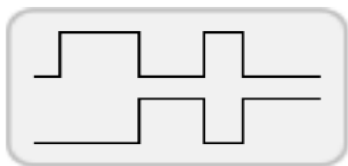

(c) Last-fired

Figure 2. Different feature representations [8]. 


\subsection{Feature insertion}

In this work, we improve the classification performances of class activities by introducing the feature insertion stage. We added two new features to the existent data matrix. The first attribute corresponds to the hour of beginning of the activity. We extract this feature directly from the data structure. The sensor activations are collected by the state-change sensors distributed all around the environment. To find out the second feature corresponding to the room label of performed activity, we search the different objects he is manipulating in the sensors, see the below figure. ID: is a number representing the sensor ID. Each sensor has its own unique ID.

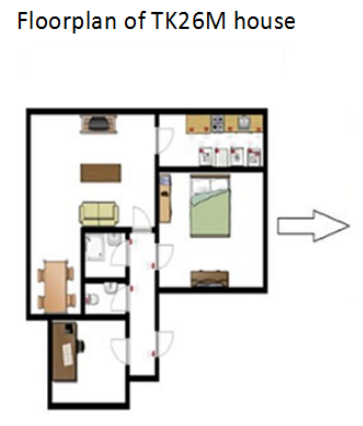

\begin{tabular}{|c|c|}
\hline \multicolumn{2}{|c|}{ Data structure } \\
\hline Start time & End time \\
\hline 25-Feb-2008 00:20:14 & 25 -Feb-2008 00:22:57 \\
\hline 25-Feb-2008 09:33:41 & 25-Feb-2008 09:33:42 \\
\hline 25-Feb-2008 09:33:47 & 25-Feb-2008 09:35:12 \\
\hline 25-Feb-2008 09:36:43 & $25-F e b-2008$ 09:37:04 \\
\hline 25-Feb-2008 09:37:20 & $25-F e b-2008 \quad 09: 37: 23$ \\
\hline 25-Feb-2008 09:37:51 & 25-Feb-2008 09:37:52 \\
\hline 25-Feb-2008 09:37:55 & 25-Feb-2008 09:37:56 \\
\hline 25-Feb-2008 09:37:58 & 25-Feb-2008 09:38:01 \\
\hline 25-Feb-2008 09:49:27 & 25-Feb-2008 09:49:28 \\
\hline 25-Feb-2008 09:49:31 & 25-Feb-2008 09:49:38 \\
\hline
\end{tabular}

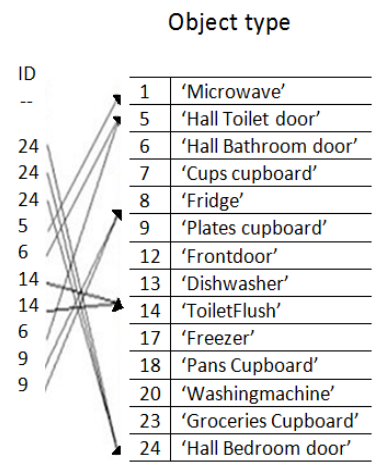

Figure 3. TF and SF for TK26M dataset. In red, the hour of beginning activity.

\subsection{Weighted Support Vector Machines Classification (WSVM)}

Osuna et al [9] proposed a Weighted SVM (WSVM) algorithm by introducing two different cost parameter $C_{\text {- }}$ and $C_{+}$in SVM optimization problem for the minority $\left(d_{i}\right.$ $=-1)$ and majority classes $\left(d_{i}=+1\right)$, as follow

$$
\begin{aligned}
& \min _{s, b, \zeta} \frac{1}{2} s \bullet s+C_{+} \sum_{i \mid \mathrm{d}_{i}=1}^{l_{+}} \zeta_{\mathrm{i}}+C_{-} \sum_{i \mid \mathrm{d}_{i}=-1}^{l_{-}} \zeta_{i} \\
& \text { subject to } d_{i}\left(\mathrm{~s} \bullet \Phi\left(y_{i}\right)+b\right) \geq 1-\zeta_{i}, \zeta_{i} \geq 0, i=1, \ldots, l
\end{aligned}
$$

$l_{+}$(resp. $l_{-}$) the number of positive (resp. negative) instances in the database. Solving the formulation dual of WSVM [5] gives a decision function for classifying a test point $y \in R^{p+q}$

$$
\mathrm{f}(x)=\operatorname{sgn}\left(\sum_{i=1}^{l_{s v}} \alpha_{i} d_{i} K\left(x, x_{i}\right)+b\right)
$$

We used the Gaussian kernel as follows: $K(x, y)=\exp \left(-\|x-y\|^{2} / 2 \sigma^{2}\right)$. Huang et al. [10] raised a Weighted SVM algorithm. The cost coefficients are typically chosen as:

$$
\frac{C_{+}}{C_{-}}=\frac{l_{-}}{l_{+}}
$$


To extend Weighted $S V M$ to the multi-class scenario in order to deal with $N$ classes (daily activities), we used different misclassification $C_{i}$ per class similar to [11]. By taking $C_{-}=C_{i}$ and $C_{+=} C$, with $l_{+}$and $l_{\mathrm{i}}$ be the number of samples of majority classes and number of samples in the $i^{\text {th }}$ class and $C$ is the common cost parameter of the WSVM. The main ratio cost value $C_{i}$ for each activity can be obtained by:

$$
C_{i}=\operatorname{round}\left(C \times\left[l_{+} / l_{\mathrm{i}}\right]\right) \quad i=1, \ldots, N
$$

\section{Simulation results and Assessment}

\subsection{Datasets}

We used fully labeled datasets [1], [3], [7] gathered by a single occupant from four houses having different layouts. We chose the ideal time slice length for discretizing the sensor data $\Delta \mathrm{t}=60$ seconds. We splitted the initial dataset into training and testing subsets using the 'leave one day out' approach, retaining one full day of sensor readings for testing and using the remaining sub-samples as training data.

\subsection{Results}

We optimized the SVM hyper-parameters $(\sigma, C)$ for all training sets in the range $[0.1-2]$ and $\{0.1,1,5,10,100\}$, respectively, to maximize the error rate of leaveoneday-out cross-validation technique. The number of features after extraction for PCA and LDA is mentioned in [5]. Then, for WSVM classification method, we optimized locally the cost parameter $C_{i}$ adapted to different classes.

In table 1 , the results show that the feature insertion set using either TF or SF contributes to significantly enhance the performance of PCA-LDA-WSVM classifier. One also notices that the TF is slightly better than SF for recognizing activities.

Table 1. Recall, Precision, F-measure and Accuracy results for all approaches in (\%). Bold values are the results for our approaches for each dataset.

\begin{tabular}{c|c|cccc}
\hline Datasets & Approach & Recall & Precision & F-Measure & Accuracy \\
& & & & & \\
\hline \multirow{3}{*}{ TK26M } & PCA-LDA-WSVM [5] & 78.8 & 80.1 & 79.4 & 95.6 \\
& TF-PCA-LDA-WSVM & $\mathbf{8 2}$ & $\mathbf{8 2 . 8}$ & $\mathbf{8 2 . 4}$ & $\mathbf{9 3 . 8}$ \\
& SF-PCA-LDA-WSVM & $\mathbf{8 0 . 4}$ & $\mathbf{8 3 . 4}$ & $\mathbf{8 1 . 8}$ & $\mathbf{9 4 . 7}$ \\
\hline TAP80F & PCA-LDA-WSVM [5] & 41.4 & 49.6 & 45.1 & 75.8 \\
& TF-PCA-LDA-WSVM & $\mathbf{4 3 . 2}$ & $\mathbf{5 1 . 3}$ & $\mathbf{4 6 . 9}$ & $\mathbf{6 5 . 4}$ \\
& SF-PCA-LDA-WSVM & $\mathbf{4 4 . 7}$ & $\mathbf{4 6 . 8}$ & $\mathbf{4 5 . 7}$ & $\mathbf{7 0 . 7}$ \\
\hline OrdonezA & PCA-LDA-WSVM [5] & 65.0 & 71.7 & 68.2 & 88.4 \\
& TF-PCA-LDA-WSVM & $\mathbf{6 8 . 7}$ & $\mathbf{7 5 . 2}$ & $\mathbf{7 1 . 8}$ & $\mathbf{8 4 . 1}$ \\
& SF-PCA-LDA-WSVM & $\mathbf{6 8 . 0}$ & $\mathbf{7 3 . 0}$ & $\mathbf{7 0 . 4}$ & $\mathbf{8 6 . 5}$ \\
\hline
\end{tabular}

We report in figure 4, the classification results in terms of accuracy measure for each class. In TK26M dataset, our proposed combinations outperforms the other 
approaches for 'Idle', 'Leaving', 'Toileting', 'Showering', 'Breakfast', 'Dinner' and 'Drink' activities. The majority activities are better for all methods over all datasets while the 'Idle' activity is more accurate for the proposed method compared to other methods. Additionally, the kitchen-related activities as 'Breakfast', 'Dinner' and 'Drink' are in general harder to recognize than other activities.

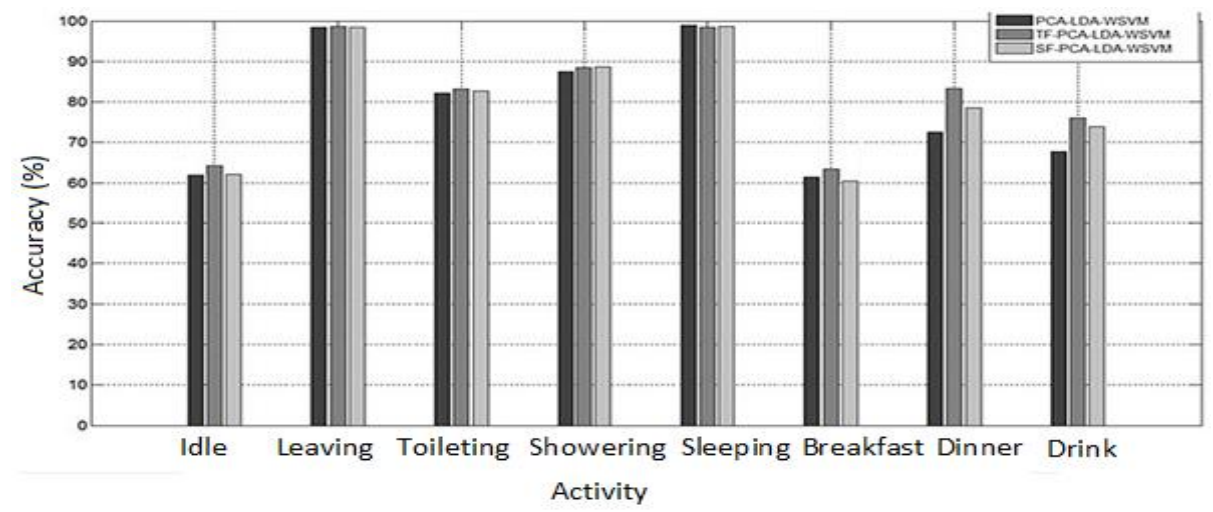

Figure 4. Accuracy recognition rate for each activity on TK26M dataset.

In order to quantify the extent to which one class is harder to recognize than another one, we analyzed the confusion matrix of TF-PCA-LDA-WSVM for TK26M dataset in Table 2. One notices that the activities 'Leaving', 'Toileting', 'Showering', 'Sleeping', 'Dinner' and 'Drink' are better recognized comparatively with 'Idle' and 'Breakfast'. The kitchen activities seem to be more recognized using the proposed method combining TF with the PCA-LDA-WSVM classifier.

Given the considerations pointed out previously, the high performance obtained in the case of TK26M dataset, which seems to be less vulnerable to class-overlapping than others, as compared to other datasets. This overlapping between the activities is due to the layout of the house. In the TK26M House, there is a separate room for almost every activity. The kitchen activities are food-related tasks, they are worst recognized because most of the instances of these activities were performed in the same location (kitchen) using the same set of sensors. Therefore the location of sensors strongly influences recognition performance.

Table 2. Confusion matrix (values in \%) of activities for TF-PCA-LDA-WSVM on the TK26M dataset.

\begin{tabular}{cllllllll}
\hline Activity & Id & Le & To & Sh & Sl & Br & Di & Dr \\
\hline Id & $\mathbf{6 4 . 3}$ & 7.6 & 2.0 & 1.1 & 4.7 & 8.7 & 6.5 & 5.1 \\
\hline Le & 0.6 & $\mathbf{9 8 . 6}$ & 0.2 & 0.5 & 0.0 & 0.0 & 0.1 & 0.0 \\
\hline $\mathrm{To}$ & 7.8 & 5.2 & $\mathbf{8 3 . 2}$ & 2.1 & 0.7 & 0.6 & 0.1 & 0.3 \\
\hline $\mathrm{Sh}$ & 5.2 & 0.0 & 4.2 & $\mathbf{8 8 . 5}$ & 0.0 & 0.0 & 0.9 & 0.2 \\
\hline $\mathrm{Sl}$ & 0.3 & 0.3 & 0.4 & 0.5 & $\mathbf{9 8 . 5}$ & 0.0 & 0.0 & 0.0 \\
\hline $\mathrm{Br}$ & 16.0 & 0.0 & 0.9 & 0.2 & 0.6 & $\mathbf{6 3 . 4}$ & 11.6 & 7.3 \\
\hline $\mathrm{Di}$ & 5.0 & 0.7 & 0.3 & 0.0 & 0.5 & 2.6 & $\mathbf{8 3 . 4}$ & 3.5 \\
\hline $\mathrm{Dr}$ & 6.8 & 0.0 & 0.0 & 0.2 & 0.2 & 6.2 & 1.7 & $\mathbf{7 6 . 1}$ \\
\hline
\end{tabular}




\section{Conclusion}

Our experiments on real-world datasets from smart home environment showed that the strategy (TF or SF)-PCA-LDA-WSVM can significantly increase the recognition performance to classify multiclass sensory data, and can improve the prediction of the minority activities. It significantly outperforms the results of the typical methods PCA-LDA-HMM and PCA-LDA-WSVM. TF-PCA-LDA-WSVM is slightly better than SF-PCA-LDA-WSVM. We added the space features needs a prior knowledge about the smart home, which makes a model very specific for that environment.

\section{References}

[1] Tapia, E.M., Intille, S.S., and Larson, K. Activity recognition in the home using simple and ubiquitous sensors, In Proc. Pervasive Computing, Lectures Notes in Computer Science Volume 3001, Vienna, pp. 158-175, 2004.

[2] Logan, B., Healey, J., Philipose, M., Tapia, EM., Intille S. A long-term evaluation of sensing modalities for activity recognition. In: Proceedings of the 9th international conference on ubiquitous computing. Springer, Berlin, pp 483-500, 2007.

[3] Ordonez, FJ., de Toledo, P., Sanchis, A. Activity recognition using hybrid generative/discriminative models on home environments using binary sensors. Sensors 13:5460-5477, 2013.

[4] Abidine, M.B., Fergani, L., Fergani, B., \& Fleury, A. Improving Human Activity Recognition in Smart Homes. International Journal of E-Health and Medical Communications (IJEHMC), 6(3), 19-37, 2015.

[5] Abidine, M.B., Fergani, L., Fergani, B., Oussalah, M. The joint use of sequence features combination and modified weighted SVM for improving daily activity recognition. Pattern Analysis and Applications, Springer-Verlag London, August 2016.

[6] Fleury, A., Noury, N. et Vacher, M. Improving Supervised Classification of Activities of Daily Activities of Daily Living Using Prior Knowledge. International Journal of E-Health and Medical Communications, Vol. 2(1), pp. 17-34, Jan-Mar, 2011.

[7] Kasteren, T.V., Noulas, A., Englebienne, G., and Krose, B. Accurate activity recognition in a home setting, UbiComp '08, ACM, New York, NY, pp. 1-9, 2008.

[8] Kasteren, T.V., Activity recognition for health monitoring elderly using temporal probabilistic models. Ph.D. thesis, University of Amsterdam, Amsterdam, The Netherlands, 27 April 2011.

[9] Osuna, E., Freund, R., Girosi, F. Support vector machines: training and applications. Technical Report. Massachusetts Institute of Technology, Cambridge, MA, USA, 1997.

[10] Huang, YM., Du, SX. Weighted support vector machine for classification with uneven training class sizes. In: Proceedings of the IEEE international conference on machine learning and cybernetics, vol 7, pp 4365-4369, 2005.

[11] Chen, D. R., Wu, Q., Ying, Y., \& Zhou, D. X. Support vector machine soft margin classifiers: error analysis. Journal of Machine Learning Research, 5(Sep), 1143-1175, 2004. 